\title{
Microsatellite-based Paternity Analysis in Polyploid Sweetpotato
}

\author{
M.I. Buteler ${ }^{1}$ and D.R. LaBonte ${ }^{2}$ \\ Department of Horticulture, Louisiana State University Agricultural Center, Louisiana Agricultural \\ Experiment Station, Baton Rouge, LA 70803 \\ R.L. Jarret \\ U.S.Department of Agriculture-AgriculturalResearch Service, Plant Genetic Resources, 1109 Experiment \\ Street, Griffin, GA 30223 \\ R.E. Macchiavelli \\ Department of Agronomy and Soils, University of Puerto Rico, P.O. Box 9030, Mayaguez, PR 00681
}

ADDITIONAL INDEX WORDS. molecular markers, genealogy reconstruction, paternity exclusion, most-likely parent, Ipomoea batatas

\begin{abstract}
AвsTRACT. Using codominant molecular markers (microsatellites) for paternity identification was investigated in hexaploid sweetpotato [Ipomoea batatas (L.) Lam.]. Two experimental populations (CIP and LAES), each consisting of progeny of known parentage, were scored for the presence or absence of alleles segregating at IB-316 and IB-318 microsatellite loci. Paternity was assessed using paternity exclusion and the most-likely parent methods. In the former, paternity is assigned based on the identification of incompatible parent-progeny marker data. In contrast, the latter method incorporates paternity exclusion and a log-likelihood or LOD score that weighs progeny allelic patterns as to the likelihood that they could have come from a given paternal parent. The number of correctly allocated progeny differed for the methods. Paternity exclusion correctly allocated $7 \%$ and $25 \%$ of the progeny in the LAES and CIP populations, respectively. The most-likely parent method correctly allocated $23 \%$ and $88 \%$ of the progeny in the LAES and CIP populations, respectively. The greater misassignments in the LAES population were attributed to low allelic diversity at the LAES IB-318 locus and a larger parental population. This study demonstrates the feasibility of identifying paternity in sweetpotato using a minimal number of loci.
\end{abstract}

Breeding programs ideally seek to identify parents that combine and generate a high number of superior progeny. The term combining ability defines this concept (Sprague and Tatum, 1942) and denotes how well a parent performs in producing superior progeny when crossed with many other parents [general combining ability (GCA)] or with specific individuals [specific combining ability (SCA)]. Procedures that enable the identification of desirable parental combinations are likely to facilitate the development of superior cultivars.

Systematically identifying desirable parental combinations requires 1) crossing all possible parents amongst themselves in a diallel cross, and 2) evaluating progeny for fitness for a given trait or in totality for all important attributes. Corn (Zea mays L.) has served as a theoretical and practical model for determination of GCA- and SCA-based crop improvement. Its ease of crossing, genetically uniform hybrid progeny, and lack of incompatibility barriers are a few of the reasons why combining ability tests are routinely used in corn improvement programs. In contrast, sweetpotato (Ipomoea batatas) is poorly suited for SCA-based improvement. This crop has complex self- and cross-incompatibility barriers (Jones et al., 1986; Martin, 1982) that often prevent the successful hybridization of selected parents. Hence, sweetpotato breeding programs rely on the use of open-pollinated

Received for publication 1 Oct. 2001. Accepted for publication 5 Feb. 2002. Approved for publication by the director of the Louisiana Agricultural Experiment Station as manuscript number 01-28-0156. This research was funded in part by the Louisiana Board of Regents, Louisiana Education Quality Support Fund contract no. LEQSF(96-98)RD-A-02 and the Louisiana Sweet Potato Advertising and Development Commission, and by state and matching funds allocated to the Louisiana State University Agricultural Center. We gratefully acknowledge Dapeng Zhang (International Potato Center) for the CIP experimental population. ${ }^{1}$ Current address: Universidad Empresarial Siglo 21, Rondeau 165, Córdoba (5000), Argentina

${ }^{2}$ Corresponding author; e-mail dlabonte@agctr.lsu.edu. (polycross) nurseries to generate progeny. While the maternal parent in this type of hybridization procedure is easily identified, the paternal parent could potentially be any one of the 12 to 15 heterozygous pollen parents in the nursery. The only way to identify parents exhibiting SCA is to hand-cross-selected parents, generate the subsequent generation from seed, clonally increase the progeny, and evaluate the progeny for desirable characteristics. Few sweetpotato breeding programs, with the exception of the Japanese and Chinese programs (Yamakawa, 1989), can afford to evaluate lines for combining ability by hand-crossing. Yet, targeting specific parental crosses exhibiting SCA would significantly enhance the efficiency of sweetpotato breeding programs. We have found that the majority of the superior progeny retained in the LSU AgCenter sweetpotato breeding program can be traced to two or three of the original maternal parents. We speculate that paternity may be similarly limited to only a few lines. The identification of superior lines a priori, is seen as a means to increase the efficacy of the polycross nursery.

Two basic approaches are typically used to establish paternity; both were developed for human population studies and forensic applications, and are still not universally accepted in their entirety (Chakraborty et al., 1974; Thompson 1975, 1986; Valentin, 1980). The first approach, referred to as paternity exclusion, is based on near conclusive proof of nonpaternity as determined by parent-offspring marker genotype data (Chakraborty et al., 1988). Paternity exclusion compares the progeny genotype with the maternal genotype, subtracts the maternal contribution, and compares the remaining paternal gametic contribution with all putative paternal genotypes. The individuals that cannot provide the observed paternal gametic contribution are excluded, and paternity is assigned to the individual that remains. In large natural mating populations, the true paternal parent cannot be assigned with $100 \%$ certainty (Chakraborty et al., 1988). The second 
approach, referred to as the most-likely parent method, calculates paternity likelihood based on segregation (Mendelian) probabilities. Parentage is assigned to the putative parent with the maximum (numerically highest) log-likelihood or LOD score. In essence, progeny alleles (genetic markers) for a given locus are weighted (based on their relative frequency in the parental population) as to the likelihood that they could have come from a given paternal parent. The more alike the two genetic marker patterns are, the higher the LOD score for the paternal parent. For example, an allele $\mathrm{D}$ that is scarce in a parental population would be uniquely invaluable in matching a lone parent and all progeny possessing this allele. In the case of tied LOD scores, no parent is selected. This approach allows paternity assignment to a higher number of progeny than paternity exclusion (Devlin et al., 1988; Smouse and Meagher, 1994). Caveats and details about these approaches are discussed in Buteler et al. (1997).

The characteristics of a genetic marker that make it suitable for paternity analysis are as follows: 1) the marker should be unambiguously inherited, 2) it should segregate independently in the population, and 3) it should lead to lower levels of ambiguity than the parentage uncertainty to be solved (Smouse and Meagher, 1994). Ideal markers for plant paternity analysis would disclose multiple, codominant alleles, be uniformly distributed throughout the genome, easily differentiate genetically similar individuals, and be easy to generate and score. Simple Sequence Repeats (SSR) (Jacob et al., 1991) or microsatellites (Litt and Luty, 1989) represent such a class of markers. In short, microsatellite loci consist of varying numbers of tandemly repeated di-, tri-, or tetranucleotide DNA motifs. They exhibit Mendelian segregation and codominance-characteristics well suited for paternity analysis (Cregan et al., 1994; Jarret and Bowen, 1994). SSR markers have been successfully applied on animal and plant diploid populations to determine genealogy structure and kin relationships (Adato et al., 1995; Morin et al., 1994; Saghai Maroof et al., 1994). There are no known data relative to the use of SSR for parentage assignment in plants.

The objective of this study was to assess paternity exclusion and most-likely parent methods for paternity assignment in hexaploid sweetpotato using microsatellites.

\section{Materials and Methods}

Plant material. Two groups of plant material were used. The first group consisted of eight parents ('Resisto', 86-33, NC-C75, 90-223, 91-153, 'Excel', 'Beauregard', and 80-62) from a population from Louisiana Agricultural Experiment Station (LAES), North Carolina Agricultural Experiment Station, and USDA, U.S. Vegetable Laboratory breeding lines (LAES population). This group also contained 14 progenies derived from controlled crosses among these parents.

The second group consisted of seven parents (Nacional, Huarmeyano, ST87.006, LM87-0045, DLP886, SR68.075, and Jewel) from a genetically diverse population obtained from the International Potato Center (CIP), Lima, Peru (CIP population). This group contained eight progenies derived from controlled crosses among these parents.

Microsatellite Loci. Buteler et al., (1999) previously reported on the derivation of loci IB-316 and IB-318 and the results of Chi-square goodness-of-fit tests for segregation ratios in these two segregating populations. In short, 1) these loci showed tetrasomic inheritance patterns; and 2) loci IB-316 and IB-318 have four and six alleles, respectively. The nucleotide sequences of primer pairs that amplify these loci are listed in Table 1.

DNA EXTRACTION. DNA was extracted from $\approx 0.25 \mathrm{~g}$ of freezedried leaf tissue using a CTAB protocol (Jarret and Austin, 1994) with modifications (Colosi and Schaal, 1993). Crude DNA extracts were further purified by repeated extraction with phenolchloroform and chloroform-isoamyl alcohol. DNA was resuspended in TE.

PCR PROFILING. PCR reactions were performed in $25-\mathrm{mL}$ volumes containing $2.5 \mathrm{~mL}$ of $10 \times$ reaction buffer (Promega Corp., Madison, Wis.), $100 \mathrm{~mm}$ of each dNTP, $2 \mathrm{~mm}$ of $\mathrm{MgCl}_{2}, 1.3$ $\mathrm{U}$ of Taq polymerase in storage buffer A (Promega Corp., Madison, Wis.), $30 \mathrm{ng}$ of template DNA, $0.8 \mathrm{~mm}$ of each forward and reverse primer.

The conditions for PCR were as follows: 2 min denaturation at $95^{\circ} \mathrm{C} ; 5$ cycles of $1 \mathrm{~min}$ at $94^{\circ} \mathrm{C} ; 1 \mathrm{~min}$ at $65^{\circ} \mathrm{C}$ for primer IB$316,1 \mathrm{~min}$ at $63{ }^{\circ} \mathrm{C}$ for IB-318; and $1 \mathrm{~min}$ at $72{ }^{\circ} \mathrm{C} ; 10$ cycles of $1 \mathrm{~min}$ at $94{ }^{\circ} \mathrm{C} ; 1 \mathrm{~min}$ at $64{ }^{\circ} \mathrm{C}$ for primer IB-316, 1 min at $62^{\circ} \mathrm{C}$ for primer IB-318; and $1 \mathrm{~min}$ at $72^{\circ} \mathrm{C}$; and 25 cycles in which the denaturation conditions were $1 \mathrm{~min}$ at $90^{\circ} \mathrm{C}$ while the annealing and extension ( $1 \mathrm{~min}$ ) temperature remained unchanged from the previous cycles. All reactions contained a terminal elongation step of $72{ }^{\circ} \mathrm{C}$ for $7 \mathrm{~min}$. These conditions were programmed in a GeneAmp PCR System 9600 (Perkin-Elmer Corp., Foster City, Calif.). The amplified DNA fragments (2-mL samples) were resolved by electrophoresis in $6.5 \%$ nondenaturing polyacrylamide gels $(0.4 \mathrm{~mm}$ thick, $38.5 \mathrm{~cm}$ long) with $1 \times$ TBE buffer $(89$ mu Tris-borate and $2 \mathrm{~mm}$ EDTA, $\mathrm{pH} 8.0$ ). The gels were run at 65 $\mathrm{W}$ for $2 \mathrm{~h}$. Three lanes with $f \mathrm{X} 174 \mathrm{Hinf}$ I markers (Promega Corp., Madison, Wis.) were included. The gels were stained with silver nitrate following the procedure of Bassam et al. (1991) as modified by $\mathrm{He}$ et al. (1994). The parent and progeny genotypic profiles were then scored.

Paternity analysis. An algorithm performed the paternity analysis based on LOD scores. The algorithm has two principal routines; the first one excludes all the putative parents that show genetic incompatibility with each offspring. To perform this, all possible gametes from the female (known) and male progenitors are extracted from the respective genotypes; the female contribution is subtracted from the offspring genotype and the remainder is compared with the putative male parent contribution. The second routine calculates the likelihood of paternity for each nonexcluded putative male parent. The two algorithms were programmed in FORTRAN (Microsoft FORTRAN $\backslash$ Power Station 1993) to be run on a PC.

In a polycross nursery only two genealogical situations need to be considered: 1) relationship $\mathrm{A}-\mathrm{F}$ is the female parent of $\mathrm{O}$ and $\mathrm{M}$ is unrelated; and 2) relationship $\mathrm{B}-$ both, $\mathrm{F}$ and $\mathrm{M}$, are parents of O. The LOD score for a parent pair is (Meagher, 1986; Thompson and Meagher, 1987):

$\mathrm{L}\left(\mathrm{B} \mid \mathrm{g}_{\mathrm{o}}, \mathrm{g}_{\mathrm{f}}, \mathrm{g}_{\mathrm{m}}\right)=\sum_{\text {loci }} \log _{\mathrm{e}}\left[\frac{\mathrm{P}\left(\mathrm{g}_{\mathrm{o}} \mid \mathrm{g}_{\mathrm{f}}, \mathrm{g}_{\mathrm{m}}\right)}{\mathrm{P}\left(\mathrm{g}_{\mathrm{o}} \mid \mathrm{g}_{\mathrm{f}},-\right)}\right]=\sum_{\text {loci }} \log _{\mathrm{e}}\left[\frac{\mathrm{M}\left(\mathrm{g}_{\mathrm{o}} \mid \mathrm{g}_{\mathrm{f}}, \mathrm{g}_{\mathrm{m}}\right)}{\mathrm{M}\left(\mathrm{g}_{\mathrm{o}} \mid \mathrm{g}_{\mathrm{f}},-\right)}\right]$

where $P\left(g_{i}\right)$ is the probability of genotype $g_{\mathrm{i}}$ in a random mating population and $M$ the Mendelian probability. The symbols, $g_{o}, g_{f}$ and $g_{m}$, represent the offspring (progeny), female parent and male parent, respectively. This algorithm calculates: 1) the probability that the genotype of a given locus [e.g., the probability based on Mendelian frequencies that $A_{1} A_{3}$ in a 3 allele $\left(A_{1} A_{2} A_{3}\right)$ diploid locus model] occurs in progeny given the set of parental genotypes mating at random $\left[P\left(g_{o \Omega} g_{f}, g_{m}\right)\right]$; and 2$)$ the probability that the known maternal parent (e.g., $\mathrm{A}_{1} \mathrm{~A}_{2}$ ) passes on its essential allele found in the progeny (i.e., $\left.\mathrm{A}_{1}\right)\left[P\left(g_{o \Omega} g_{f},-\right)\right]$. In a breeding 
nursery the hypothesized relationship is B because A is known, so it can be used as the base-point alternative. To compare the likelihoods of distinct relationships (A and $\mathrm{B}$ ) the difference in the natural logarithm of the likelihoods is considered (LOD scores). Because offspring's loci are independent conditionally on the parental genotypes, LOD scores are summed across all loci.

Even though this approach allows paternity assignment to a higher number of progeny, it presents two limitations. First, categorical assignments are not possible for all the progeny for ambiguous progeny genotypic profiles. Second, a statistical bias in favor of homozygotes for a homozygous putative parent will always result in a higher LOD score for a given locus than a heterozygous individual (Devlin et al., 1988; Smouse and Meagher, 1994). Both limitations can be overcome by increasing the number of genetic markers.

Allele frequencies and gene diversity or average heterozygosity $(H)\left(\mathrm{H}=1-\sum_{\mathrm{i}=1}^{\mathrm{N}} \mathrm{p}_{\mathrm{i}}^{2}\right)$ for each population were calculated according to Nei (1987). $H$, as a measure of degree of genetic variability, is the probability that two randomly chosen alleles with frequencies $p_{i}$, the population frequency for the $i$ th allele, are different. A higher $H$ value represents a higher expected frequency of heterozygous individuals (Ott, 1992).

\section{Results}

Allele Frequencies. In the LAES parental population, five microsatellite alleles were detected in parents for locus IB-318 (Table 1). Allele frequencies in the parent population were skewed towards the $120 \mathrm{bp}$ allele. This allele, at a $75 \%$ frequency, and the $114 \mathrm{bp}$ allele accounted for $90 \%$ of the allelic population. This poor allelic diversity in progeny is reflected in a gene diversity or average $(H)$ of 0.41 . Four alleles were detected in parents for locus IB-316 (Table 1). The gene diversity was higher $(H=0.68)$, reflecting a more equitable balance in allele frequency within the parental population.

In the CIP parental population, four microsatellite alleles were detected in parents for locus IB-318 (Table 1). Allele frequencies in this parent population were again dominated by the 120 bp allele ( $54 \%$ frequency), but to a lesser extent than that found within the LAES population; concomitantly, gene diversity was correspondingly higher $(H=0.63)$. Four alleles were detected in parents for locus IB-316. Gene diversity was high and comparable to locus IB316 for the LAES progeny population. All alleles fit within the expected class size for the two loci (Buteler et al., 1999).

Paternity allocation. In the LAES population, two out of 13 progeny were correctly allocated to the paternal parent by paternity exclusion (progeny 8 and 9, Table 2). All potential parents, except for the true paternal parent showed allelic incompatibility. Most-likely parent analysis (Table 2) correctly allocated one additional progeny (10) to the true paternal parent. Ambiguous assignments, i.e., two parents with identical LOD scores, occurred for two additional progeny (3 and 13). Misassignments were made for the remaining eight progeny. Of these, the true paternal parent had the second highest LOD score for three progeny. Four progeny were similarly narrowed to within three potential paternal parents. The parent $91-153$ had six misassignments associated with it; five were traceable to parents with similar ancestry to 91-153.

In the CIP population, two out of eight progeny were correctly allocated to the paternal parent by paternity exclusion (progeny 3 and 4, Table 3). Most-likely parent analysis correctly allocated five additional progeny to the true male parent. Only one progeny was narrowly misassigned.

\section{Discussion}

Paternal allocation by the most-likely parent method was more successful when the CIP population was used as compared to the LAES population, i.e., seven out of eight progeny were allocated to the correct paternal parent vs. three out of 12 for the LAES population. The main difference between the data sets is the low level of allelic diversity at the LAES IB-318 locus. In contrast, allelic diversity (even distribution) was high for both loci in the CIP progeny population. These results are consistent with our expectations that the LAES population has a narrower genetic base. He et al. (1995) estimated that most cultivars within the United States could be traced to several common ancestors; by implication, we assume greater homogeneity among LAES parents. The CIP population, however, consists of parents from the United States and South America. In this more genetically diverse population, the informativeness of the two microsatellite loci was sufficient to determine the paternity. However, we would expect the CIP population to behave more like the LAES population as homogeneity increases under intense selection. These data sup-

Table 1. Sweetpotato microsatellite loci, allele frequencies (AFs), and heterozygosity in CIP and LAES populations for the 2 primer pairs that produce scorable bands.

\begin{tabular}{|c|c|c|c|c|c|c|c|c|}
\hline \multirow{2}{*}{$\begin{array}{l}\text { Locus } \\
\text { designation }\end{array}$} & \multirow{2}{*}{$\begin{array}{l}\text { Primer pairs } \\
5^{\prime} \ldots \ldots \ldots \ldots \ldots \ldots \ldots \ldots \ldots \ldots \ldots \ldots \ldots \ldots \ldots \ldots \ldots \ldots \ldots \ldots \ldots \ldots \ldots \\
\end{array}$} & \multirow[b]{2}{*}{ Repeat } & \multirow{2}{*}{$\begin{array}{c}\text { Expected } \\
\text { Size } \\
{[b p]^{\mathrm{z}}}\end{array}$} & \multirow{2}{*}{$\begin{array}{c}\text { Size } \\
\text { classes } \\
{[\mathrm{bp}]}\end{array}$} & \multicolumn{2}{|c|}{$\begin{array}{c}\text { CIP } \\
\text { population } \\
\end{array}$} & \multicolumn{2}{|c|}{$\begin{array}{c}\text { LAES } \\
\text { population }\end{array}$} \\
\hline & & & & & $\mathrm{H}^{\mathrm{y}}$ & $\mathrm{AF}$ & $\mathrm{H}$ & $\mathrm{AF}$ \\
\hline \multirow[t]{4}{*}{$\overline{\mathrm{IB}}-316$} & CAAACGCACAACGCTGTC & $(\mathrm{GA})_{3} \mathrm{G}(\mathrm{GA})_{8}$ & 150 & 146 & 0.68 & 0.2500 & 0.68 & 0.4038 \\
\hline & CGCGTCCCGCTTATTTAAC & & & 142 & & 0.1429 & & 0.0385 \\
\hline & & & & 138 & & 0.1429 & & 0.2692 \\
\hline & & & & 133 & & 0.4643 & & 0.2885 \\
\hline \multirow[t]{6}{*}{ IB-318 } & AGAACGCATGGGCATTGA & $(\mathrm{GA})_{9} \mathrm{G}(\mathrm{GA})_{5}$ & 124 & 120 & 0.63 & 0.5357 & 0.41 & 0.7500 \\
\hline & CCCACCGTGTAAGGAAATCA & & & 114 & & 0.1786 & & 0.1538 \\
\hline & & & & 111 & & 0.2143 & & 0.0000 \\
\hline & & & & 119 & & 0.0714 & & 0.0385 \\
\hline & & & & 123 & & & & 0.0385 \\
\hline & & & & 125 & & & & 0.0192 \\
\hline
\end{tabular}

\footnotetext{
${ }^{\mathrm{z}}$ Band size in base pairs.

${ }^{\mathrm{y}}$ Measure of degree of polymorphism, expected heterozygosity.
} 
Table 2. LOD score matrix for paternity allocation in the LAES population for loci IB-316 and IB-318.

\begin{tabular}{|c|c|c|c|c|c|c|c|c|}
\hline \multirow[b]{2}{*}{ Parents } & \multicolumn{8}{|c|}{ Offspring $^{2}$} \\
\hline & Resisto & $86-33$ & $\mathrm{NC}-\mathrm{C} 75$ & $90-233$ & $91-153$ & Excel & Beauregard & $80-62$ \\
\hline$\overline{1}$ & $0.000000^{y}$ & $-1.436441^{\mathrm{w}}$ & 0.355317 & 0.000000 & $1.453930^{\mathrm{x}}$ & -0.337829 & -0.337829 & -1.436441 \\
\hline 2 & 0.000000 & $0.08661434^{w}$ & 0.000000 & 0.000000 & ${\underline{3.063367^{x}}}^{\underline{x}}$ & 1.2716085 & 1.2716085 & 0.8661434 \\
\hline 3 & $1.721809^{w}$ & 0.000000 & ${\underline{1.721809^{v}}}^{\mathrm{v}}$ & 0.000000 & 0.000000 & 0.000000 & 1.0286623 & 0.000000 \\
\hline 4 & 0.000000 & -1.436441 & $0.355317^{w}$ & 0.000000 & ${\underline{1.453930^{\underline{x}}}}^{\underline{x}}$ & -0.337829 & -0.337829 & -1.436441 \\
\hline 5 & 0.000000 & -1.436441 & 0.355317 & 0.000000 & ${\underline{1.453930^{x}}}^{\mathrm{x}}$ & 0.337829 & $-0.337829^{w}$ & -1.436441 \\
\hline 6 & 0.000000 & -1.554224 & 0.930681 & 0.000000 & $\underline{1.336147^{\underline{x}}}$ & $0.050147^{w}$ & 0.2375347 & -1.554224 \\
\hline 7 & 0.000000 & -0.312511 & 0.000000 & 0.000000 & 1.884712 & $2.2901781^{\mathrm{w}}$ & $\underline{2.9833252^{\mathrm{x}}}$ & -0.312511 \\
\hline 8 & 0.000000 & $4.2755375^{\mathrm{w}}$ & 0.000000 & 0.000000 & 0.000000 & 0.000000 & 0.000000 & 0.000000 \\
\hline 9 & 0.000000 & 0.000000 & 0.000000 & 0.000000 & 0.000000 & 0.000000 & $2.6579028^{w}$ & 0.000000 \\
\hline 10 & 0.000000 & 0.8661434 & 0.000000 & 0.000000 & $3.063367^{w}$ & 1.2716085 & 1.2716085 & 0.8661434 \\
\hline 11 & 0.000000 & 0.000000 & 0.000000 & 0.000000 & 0.000000 & $2.6579028^{w}$ & ${\underline{3.3510500^{\mathrm{x}}}}^{-1}$ & 0.000000 \\
\hline 12 & 0.000000 & -0.520150 & 0.172996 & 0.000000 & ${\underline{1.964755^{\mathrm{x}}}}^{-}$ & 0.1729962 & $\overline{0.1729962}$ & $-0.520150^{\mathrm{w}}$ \\
\hline 13 & 0.000000 & -1.659585 & $1.230786^{\mathrm{v}}$ & 0.000000 & $1.230786{ }^{\mathrm{w}}$ & 0.1321742 & 0.5376393 & -1.659585 \\
\hline
\end{tabular}

$\bar{z}$ The higher LOD score determines parental allocation. Female parents are as follows for offspring: $1=$ Resisto, $2=$ Resisto, $3=86-33,4=$ Resisto, $5=$ NC-C75, 6 = 90-223, 7 = NC-C75, $8=$ NC-C75, $9=$ Resisto, $10=$ NC-C75, $11=$ NC-C75, $12=91-153,13=$ Excel.

yExcluded male parent $(\mathrm{LOD}=0.0)$.

${ }^{\mathrm{x}}$ Misassigned male parent (underlined).

wTrue male parents (bold).

vAmbiguous parental allocation.

port the evidence obtained by simulation studies that skewed allele frequencies (i.e., two alleles among five account for more than $90 \%$ of the alleles present at the IB-318 locus in the LAES population) increase the number of misassigments and the variability of the estimates (Buteler et al., 1997). A second difference in these data sets is parent population size. The CIP parental population was smaller than the LAES parental population, i.e., five parents vs. eight parents, respectively. Buteler et al. (1997) showed via computer simulation that misassignments increased as a function of increasingly larger parental populations.

The algorithm used in the present study allocated parental assignments based on paternity exclusion and LOD scores. Paternity exclusion correctly allocated two progeny in each population and represented a $100 \%$ confidence level, given that no other paternal parents were a part of the experimental population. Yet, the most-likely parent method correctly allocated additional progeny in the populations. The confidence that one places on accurate parentage assignment is based on the numbers of loci and alleles used in the data set. Buteler et al. (1997), using computer simulation studies with a hexaploid, showed that the number of loci scored for a 10-parent population should not be less than 20 in the case of three alleles per locus, and no more than 10 loci for a five allele per locus model for parental discrimination with negligible errors or misassignments. A three loci, five allele (even allele frequency) model is accurate to within 15\%. An incremental increase in the number of alleles enhances discriminatory power to a greater extent than an increase in the number of loci. Albeit, our present work was limited to two loci; other microsatellite loci were tested but these failed to segregate in a Mendelian fashion (Buteler et al., 1999). Microsatellite loci in sweetpotato show insertions, deletions, and base substitutions in addition to point mutations within the repeat and adjacent regions (Buteler et al., 1999). These aberrations may impact Mendelian segregation patterns and complicate discovery of new loci. Nevertheless, additional microsatellites are being developed for multiple uses in sweetpotato (Zhang et al., 2001).

In conclusion, paternity can be allocated with few loci in an experimental population. The most-likely parent method showed greater discriminatory power when compared with paternity exclusion for paternity determination in both populations. These

Table 3. LOD score matrix for paternity allocation in the CIP population for loci IB-316 and IB-318.

\begin{tabular}{|c|c|c|c|c|c|}
\hline \multirow[b]{2}{*}{ Parents } & \multicolumn{5}{|c|}{ Offspring $^{z}$} \\
\hline & Nacional & Huarmeyano & ST87.006 & LM87.045 & Jewel \\
\hline 1 & $0.000000000000^{y}$ & 0.000000000000 & -0.595754879255 & $1.483686662425^{w}$ & 0.000000000000 \\
\hline 2 & 0.000000000000 & 0.000000000000 & 0.224144006944 & 2.526729099938 & 0.000000000000 \\
\hline 3 & 0.000000000000 & 0.000000000000 & 0.000000000000 & 30.024131703372 & 0.000000000000 \\
\hline 4 & 0.000000000000 & 0.000000000000 & 0.000000000000 & 30.024131703372 & 0.000000000000 \\
\hline 5 & -0.354592822434 & $1.842631745898^{w}$ & 0.000000000000 & -1.453205111106 & 1.149484574338 \\
\hline 6 & 0.369326016789 & 2.161085486017 & 0.000000000000 & 0.000000000000 & 1.062473197349 \\
\hline 7 & 0.000000000000 & 0.908322517521 & -1.576584132267 & 0.000000000000 & $1.719252733738^{w}$ \\
\hline 8 & 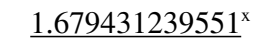 & 1.456287688237 & 0.069993327117 & 0.000000000000 & 1.574070723893 \\
\hline
\end{tabular}

The higher LOD score determines parental allocation. Female parents are as follows for offspring: $1=$ Nacional, $2=$ Nacional, $3=$ Huarmeyano, 4 = Huarmeyano, $5=$ ST87.006, $6=$ ST87.006, $7=$ Nacional, $8=$ LM87.045.

${ }^{\mathrm{y}}$ Excluded male parent $(\mathrm{LOD}=0.0)$.

${ }^{\mathrm{x}}$ Misassigned male parent (underlined).

wTrue male parents (bold). 
data sets underscore the utility of the most-likely parent method, i.e., no LOD based paternal allocation is made without first eliminating paternal parents based on paternity exclusion. Our results demonstrate the feasibility of microsatellite-based paternity analysis in polyploid species and statistical approaches to paternity determination in plant breeding programs.

\section{Literature Cited}

Adato, A., D. Sharon, U. Lavi, J. Hillel, and S. Gazit. 1995. Application of DNA fingerprints for identification and genetic analysis of mango (Mangifera indica) genotypes. J. Amer. Soc. Hort. Sci. 120:259-264.

Bassam, B.J., G. Caetano-Anolles, and P.M. Gresshoff. 1991. Electrophoresis of polyester-backed polyacrylamide gels. Anal. Biochem. 196:80 83.

Buteler, M.I., D.R. La Bonte, and R.E. Macchiavelli. 1997. Determining paternity in polyploids: Hexaploid simulation studies. Euphytica 96:353361.

Buteler, M.I., R.L. Jarret, and D.R. La Bonte. 1999. Sequence characterization of microsatellites in diploid and polyploid Ipomoea. Theor. Appl. Genet. 99:123-132.

Chakraborty, R., M. Shaw, and W.J. Schull. 1974. Exclusion of paternity: the current state of the art. Amer. J. Hum. Genet. 26:477-488.

Chakraborty, R., T.R. Meagher, and P.E. Smouse. 1988. Parentage analysis with genetic markers in natural populations. I. The expected proportion of offspring with unambiguous paternity. Genetics 118:527-536.

Colosi, J.C. and B.A. Schaal. 1993. Tissue grinding with ball bearings and vortex mixer for DNA extraction. Nucleic Acids Res. 21:1051-1052.

Cregan, P.B., A.A. Bhagwat, M.S. Akkaya, and J. Rongwen. 1994. Microsatellite fingerprinting and mapping in soybean. Meth. Mol. Cell Biol. 5:49-61.

Devlin, B., K. Roeder, and N.C. Ellstrand. 1988. Fractional paternity assignment: theoretical development and comparison to other methods. Theor. Appl. Genet. 76:369-380.

He, G., C.S. Prakash, R.L. Jarret, S. Tuzun, and J. Qiu. 1995. Comparison of gel matrices for resolving PCR-amplified DNA fingerprint profiles. PCR Meth. Appl. 4:50-51.

Jacob, H.J., K. Lindpaintner, S. Lincoln, K. Kusumi, R. Bunker, Y-P Mao, D. Ganten, V. Danau, and E. Lander. 1991. Genetic mapping of a gene causing hypertension in the stroke-prone hypertensive rat. Cell 67:213224.

Jarret, R.L. and D.F. Austin. 1994. Genetic diversity and systematic relationships in sweetpotato (Ipomoea batatas (L.) Lam.) and related species as revealed by RAPD analysis. Genet. Res. Crop Evol. 41:165173.

Jarret, R.L. and N. Bowen. 1994. Simple Sequence Repeats (SSRs) for sweetpotato germplasm characterization. Plant Genet Res. Nwslt. 100:9_ 11.

Jones, A., P.D. Dukes, and J.M. Schalk. 1986. Sweet potato breeding, p. 135. In: M.J. Basset (ed.). Breeding vegetable crops. AVI Publishing Co., Inc., Westport, Conn.

Litt, M. and J.A. Luty. 1989. A hypervariable microsatellite revealed by in vitro amplification of a dinucleotide repeat within the cardiac muscle actin gene. Amer. J. Hum. Genet. 44:397-401.

Martin, F.W. 1982. Analysis of the incompatibility and sterility of sweet potato, p. 275-283. In: R.L. Villareal and T.D. Griggs (eds.). Sweet potato. Proc. 1st Intl. Symp. Asian Veg. Res. Dev. Ctr., Shanhua, Taiwan. Meagher, T.R. 1986. Analysis of paternity within a natural population of Chamaelirium luteum. 1. Identification of most-likely parents. Amer. Nat. 128:199-215.

Morin, P.A., J.J. Moore, R. Chakraborty, L. Jin, J. Goodall, and D.S. Woodruff. 1994. Kin selection, social structure, gene flow, and the evolution of chimpanzees. Science 265:1193-1201.

Nei, M.1987. Molecular evolutionary genetics. Columbia Univ. Press, New York.

Ott, J., 1992. Analysis of human genetic linkage. revised ed. Johns Hopkins Univ. Press, Baltimore, Md.

Saghai Maroof, M.A., R.M. Biyashev, G.P. Yang, Q. Zhang, and R.W. Allard. 1994. Extraordinarily polymorphic microsatellite DNA in barley: Species diversity, chromosomal locations, and population dynamics. Proc. Natl. Acad. Sci. USA 91:5466-5470.

Smouse, P.E. and T.R. Meagher. 1994. Genetic analysis of male reproductive contributions in Chamaelirium luteum (L.) Gray (Liliaceae). Genetics. 136:313-322.

Sprague, G.F., and L.A. Tatum. 1942. General and specific combining ability in single crosses of corn. J. Amer. Soc. Agron. 34:923-932.

Thompson, E.A. 1975. The estimation of pairwise relationship. Ann. Hum. Genet. 39:173-188.

Thompson, E.A. 1986. Pedigree analysis in human genetics. Johns Hopkins Univ. Press, Baltimore, Md.

Thompson, E.A., and T.R. Meagher. 1987. Parental and sib likelihoods in genealogy reconstruction. Biometrics 43:585-600.

Valentin, J. 1980. Exclusions and attributions of paternity: Practical experiences of forensic genetics and statistics. Amer. J. Hum. Genet. 32:420-431.

Yamakawa, O. 1989. Annual report of sweetpotato breeding. Sweetpotato Breeding Laboratory, Kyushu Natl. Agr. Expt. Sta., Min. Agr., For. Fish., Japan.

Zhang, D.P., D. Carbajulca, L. Ojeda, G. Rossel, S. Milla, C. Herrera, and M. Ghislain. 2001. Microsatellite analysis of genetic diversity in sweetpotato varieties from Latin America, p. 295-301. In: CIP Program Rpt. 1999-2000. Intl. Potato Ctr., Lima, Peru. 